\title{
Cutting edge publishing platform in research, innovation and advancement in the field of automation and robotics
}

\section{Editorial}

International Robotics and Automation Journal (IRATJ) is a peer reviewed, scholarly and open-access journal which targets a need for investigation and growth in the field of Robotics and Automation alongside rapid technological innovation. Online Access to this Journal is open to all where the published articles are available for the readership but on condition that the usual referencing and acknowledgements are adhered to as with all academic publications. The process of publication of Online Journals is the future of knowledge sharing and publishing advances in Science and technology. This is complicated in the sense that it includes all the overheads such as editing, proofreading, quality check, web maintenance, plagiarism check and much more. The tasks included in the process of submission and publishing of the journals includes some costs and that is why the processing fees are required. In order to allow quality research to be published the charging of fees is done with sensitivity and flexibly with full cognizance of the authors' ability to pay. Peer review of the submitted articles plays a very important role in ensuring the integrity of the scholarly record for our system. The process depends to a large extent on trust, and requires that everyone involved behaves responsibly and ethically. Peer reviewers play a central and critical part in the peer-review process. We get at least two independent reviews of each full length article and at least one for short report letters. We use a double-blind reviewing process for every submission made to the journal in which author's and reviewer's identities are concealed. Reviewers of all journal content are advised to provide applicable and beneficial feedback which is geared towards enhancing the quality of work to be published. Clear distinction is made between all obligatory recommendations and others which are optional. The final decision is made by the handling editor subsequent to careful consideration of all returned reviews. Additional reviewers can be requested for cases of conflicting or vastly differing reviews.

At MedCrave ethics is considered of critical importance in both professional and personal aspects. All manuscripts are only accepted for publication pending extensive compliance checks with our ethical standards. This IRATJ is a very broadly classified publication which includes Robotic Technology, Automation, Power Sources, Actuation, Sensing, Manipulation, Locomotion, Environmental Interaction and Navigation, Human-Robot Interaction, Autonomy Levels, Dynamics and Kinematics, Types of Automation. Furthermore focus may be in design, construction, operation, and use of robots, as well as computer systems for their control, sensory feedback and information processing systems. The scope of the subjects includes industry, domestic, medical, and services, military, gaming and entertainment, space and leisure/hobby developments. Because of the complexity and broad
Volume 3 Issue 4 - 2017

\author{
Chanan S Syan \\ Department of Mechanical and Manufacturing Engineering, \\ University of the West Indies, Trinidad and Tobago
}

Correspondence: Chanan S Syan, Department of Mechanical and Manufacturing Engineering, University of the West Indies, Trinidad and Tobago, Email Chanan.Syan@sta.uwi.edu

Received: November 09, 2017 | Published: November 14, 2017

nature of our journal, it lends itself to inclusion of this fast developing and expanding area research of applied sciences and Technology.

Aspiring and continuing authors wishing to share and gain knowledge in this field benefit immensely from publishing in this journal. Successful publication allows for comparative standing of the authors work alongside similar theoretical, computational, experimental and practical aspects in the field of Automation and Robotics. Free PDFs of articles are offered as open source and contain a liberal copyright policy. The journal highly encourages articles from authors of all associations including governmental, non-profit organizations, private institutions alongside independent researchers at an international level. A PDF email of the proofs is sent to the corresponding author as well as access to a PDF of the final article inclusive of the Digital Object Identifier (DOI). Maintenance of high publication standards is of major concern in this journal and as such quality service levels are noted with regard to copy-editing, typesetting, printing and the online publication. Development of strong author relations throughout the publication process is of key importance. This journal is a highly ranked and internationally recognized journal which aims at highlighting the overall development of the Robotics and Automation field globally. I am sure that you will find this issue of IRAT journal stimulating and engaging as very relevant to the cutting edge research and developments in this fast moving area of technology.

\section{Acknowledgments}

None.

\section{Conflict of interest}

No conflict of interest. 